\title{
Originals
}

\section{Elevated GLUT 1 level in crude muscle membranes from diabetic Zucker rats despite a normal GLUT 1 level in perineurial sheaths}

\author{
A. Handberg ${ }^{1}$, L. Kayser ${ }^{2}$, P. E. Høyer ${ }^{2}$, J. Micheelsen ${ }^{1}$, J. Vinten ${ }^{1}$ \\ ${ }^{1}$ Department of Medical Physiology, Panum Institute, University of Copenhagen, Copenhagen, Denmark \\ ${ }^{2}$ Department of Medical Anatomy, Panum Institute, University of Copenhagen, Copenhagen, Denmark
}

Summary Recently, we demonstrated that approximately $60 \%$ of GLUT 1 in a crude membrane fraction of rat skeletal muscle originates from perineurial sheaths. To study the in vivo regulation of GLUT 1 expression in different tissues in muscles, we measured the level of GLUT 1 in crude muscle membranes and in perineurial sheaths in diabetic $(\mathrm{fa} / \mathrm{fa})$ Zucker rats and lean controls, with and without metformin treatment. The GLUT 1 concentration in perineurial sheaths was identical in all four groups of rats, both when measured by quantitative immunofluorescence and by immunoblotting and densitometry. In a fraction of crude membranes of soleus muscles GLUT 1 expression was more than two-fold higher in $(\mathrm{fa} / \mathrm{fa})$ rats than in lean controls $(p<0.005)$. Metformin treatment significantly elevated GLUT 1 in control rats $(p<0.05)$ and tended to decrease GLUT 1 in diabetic rats $(p<0.075)$. The expressions of GLUT 1 and GLUT 4 in crude muscle membranes were inversely correlated $(p<0.01)$, and GLUT 1 expression correlated positively with fasting glucose $(p<0.05)$. In conclusion, GLUT 1 expression in perineurial sheaths is unaffected by alterations in glucose homeostasis and by the genes responsible for obesity and diabetes in the Zucker rat. GLUT 1 expression in a crude membrane fraction of soleus muscle is increased in the diabetic animals, likely due to an increased expression in muscle cells proper. [Diabetologia (1994) 37:443-448]

Key words GLUT 4, glucose transporter, insulin resistance, (fa/fa) rats, GLUT 1, Zucker rats, skeletal muscle, perineurial sheath, crude membranes, hyperglycaemia, metformin.
Energy-independent transport of glucose across animal cell membranes is catalysed by members of the facilitative glucose transporter family (for reviews, see [1, 2]). The glucose transporter GLUT 1 is expressed in most tissues in rodents, and attains the highest concentration in the blood-brain/nerve-barrier [3-6]. The role of GLUT 1 in skeletal muscle has been subject to some discussion. In skeletal muscle from fasting rats, GLUT 1 transporters are almost exclusively located in the plasma membrane fraction and no significant effect of insulin or contraction on GLUT 1 distribution has been demonstrated [7-9]. Thus, by analogy to the situation

Received: 17 June 1993

and in revised form: 19 November 1993

Corresponding author: Dr. A. Handberg, Department of Medical Physiology, Panum Institute, Blegdamsvej 3C, DK-2200 Copenhagen, Denmark in adipocytes, it has been suggested that GLUT 1 is of particular importance for the basal glucose uptake in skeletal muscle cells. However, in other studies using immunocytochemical techniques labelling for GLUT 1 on sections of skeletal muscle was confined to the blood-brain/nerve-barrier tissues, i.e. perineurial sheaths and endoneurial vessels [10-12], and it has been proposed that the function of GLUT 1 in skeletal muscle is to mediate an adequate supply of glucose to the nerve fibres.

We have recently reported that GLUT 1 from perineurial sheaths accounts for approximately $60 \%$ of the GLUT 1 content in a crude membrane fraction from rat skeletal muscles, and that the remaining GLUT 1 is likely to represent the "constitutive" glucose transporter in the muscle cells proper [6]. In this light, interpretation and evaluation of the significance of previous results concerning GLUT 1 expression in membrane fractions of skeletal muscle in a variety of adaptive and 
pathological states is complicated by the lack of knowledge of the site of change.

Skeletal muscle of the genetically obese Zucker rat (fa/fa) is insulin resistant [13,14], and a decreased glucose transport, both in the basal and the insulin stimulated state has been demonstrated [14]. Some studies have been published concerning the expression of glucose transporters in skeletal muscle from this animal model [15-20]. However, none of these studies have specifically dealt with GLUT 1.

To acquire information about the regulation of GLUT 1 expression in perineurial sheaths and muscle membranes, we have studied the level of GLUT 1 in muscle membrane and perineurial sheath in obese Zucker rats and their lean littermates, before and after amelioration of the hyperinsulinaemia and hyperglycaemia by treatment with the oral antidiabetic drug, metformin.

\section{Materials and methods}

Treatment of animals. Obese male Zucker rats (fa/fa) and their lean littermates $(\mathrm{Fa} /-)$ were purchased from Charles River Laboratories (Cambridge, Mass., USA) at 8 weeks of age. Seven obese Zucker rats and seven lean controls were randomly chosen to be treated with metformin, and the remaining seven rats from each group served as controls. By use of a gastric tube, the rats assigned for treatment were given metformin ( N,N-dimethylbiguanid) dissolved in $0.9 \% \mathrm{NaCl}$ every morning initially at a dose of $90 \mathrm{mg} /$ day for 6 days increasing to $180 \mathrm{mg} /$ day for 6 days, $225 \mathrm{mg} /$ day for 6 days and finally $270 \mathrm{mg} /$ day for 6 days while the controls were given vehicle only. The last dose of metformin or vehicle was given $24 \mathrm{~h}$ before the day of study. The animals were weighed twice weekly. The diabetic Zucker rats were significantly more obese than their lean littermates at the onset of metformin treatment (body weight $367 \pm 16$ vs $277 \pm 6.5 \mathrm{~g}$ ). Weight gain during the study was unaffected by metformin treatment in both groups [21].

After an overnight fast, the animals were anaesthetized by i. p. injection of mebumal and weighed. The soleus muscles were excised and immediately frozen by immersion in $\mathrm{n}$-hexane at $-70^{\circ} \mathrm{C}$ and stored at the same temperature until analysis. Femoral nerves were excised, dissected free of loose connective tissue, and frozen.

Analysis of blood samples. A commercially available RIA kit was used for determination of insulin as described by the manufacturer (Novo-Nordisk, Bagsvaerd, Denmark) using rat insulin as standard, and glucose was measured by an automated glucose analyser (YSI 23 AM; Yellow Springs Instruments, Yellow Springs, Ohio, USA). Glycated fructosamine was analysed using a commercially available kit (Precimat; Boehringer Mannheim, Meylan, France), and data were normalized to concentration of plasma protein.

Immunoblotting. Frozen soleus muscles were homogenized for $7 \mathrm{~s}$ using a Polytron (max. setting) in $3 \mathrm{ml}$ of $50 \mathrm{mmol} / \mathrm{l}$ Hepes, $\mathrm{pH} 7.6,250 \mathrm{mmol} / \mathrm{sucrose}, 10 \mathrm{mmol} / \mathrm{EDTA}, 1.5 \mathrm{mmol} / \mathrm{l} \mathrm{phe}-$ nylmethylsulphonyl-flouride (PMSF) and $400 \mathrm{KIU} / \mathrm{ml}$ Trasylol. A crude membrane fraction was isolated from the homogenate by a centrifugation procedure, essentially as described in [7] with the modifications described in [22]. Protein content was deter- mined by the method of Bradford using the kit from Biorad (Richmond, Calif, USA), and protease inhibitors were added to the remains of the crude membrane preparation $(1.5 \mathrm{mmol} / \mathrm{l}$ PMSF and $400 \mathrm{KIU} / \mathrm{ml}$ Trasylol). Samples containing $50 \mu \mathrm{g}$ of protein from each crude membrane preparation were analysed in duplicate by SDS-PAGE and Western Blotting as described [22]. Protein recovery in the crude membrane preparations was identical in the four groups (data not shown).

Femoral nerves $(3.9-6.8 \mathrm{mg}$ ) were homogenized in $0.4 \mathrm{ml}$ of homogenization buffer with protease inhibitors $(1.5 \mathrm{mmol} / \mathrm{h}$ PMSF and $400 \mathrm{KIU} / \mathrm{ml}$ Trasylol) and samples of $50 \mu \mathrm{l}$ of the homogenates were analysed by SDS-PAGE and Western Blotting as described above.

Protein G-purified monoclonal antibody raised against GLUT 1 purified from human erythrocyte membranes and reacting with a peptide corresponding to the $15 \mathrm{C}$-terminal amino acids was used for detection of GLUT 1 [23]. Quantitation of immunoreactivity was performed by densitometricscanning using a preparation of adipocyte plasma membrane as internal standard.

Fluorescence labelling of GLUT 1 in tissue sections. Soleus muscles were divided into three tissue blocks by two transverse cuts at random positions on the long axis of the muscle. From each tissue block, six cryosections were cut $6 \mu \mathrm{m}$ thick and all sections from one soleus muscle were adsorbed on the same gelatin coated slide. Slides were incubated with Tris-buffered saline $(10 \mathrm{mmol} / \mathrm{l}$ Tris- $\mathrm{HCl}, \mathrm{pH} 7.4$ and $0.9 \% \mathrm{NaCl}$ ) containing $5 \%$ bovine serum albumin for $30 \mathrm{~min}$ at room temperature to block non-specific binding, essentially as previously described [6]. Triplicate sections from each tissue block (in total nine sections on each slide) were incubated for $1 \mathrm{~h}$ with monoclonal GLUT 1 antibody (see above), diluted 1: 100 in Tris-buffered saline with $1 \%$ bovine serum albumin. Duplicate sections from each tissue block (in total six sections on each slide) were incubated with dilution buffer alone and served as background sections. The cryosections were washed and incubated with BODIPY conjugated goat anti-mouse IgG antibody (B2752; Molecular Probes, Eugene, Ore., USA) for $1 \mathrm{~h}$. Finally, the sections were rinsed in Tris-buffered saline, mounted in Aquamount (BDH, Dorset, UK) and kept in the dark at $-20^{\circ} \mathrm{C}$ until image analysis.

\section{Quantitation of immunofluorescence labelling in tissue sections}

Instrumentation. The fluorescence intensity emitted from the specifically bound secondary antibody was measured using a Zeiss Axiovert 10 epifluorescense microscope equipped with a Plan Neofluar water immersion objective (Magnification $\times 16$, numerical aperture 0.50 ), a XBO $75 \mathrm{~W}$ light source, a fast-shutter and a Dage/MTI SIT 66 camera (Michigan City, Ind.,USA) connected to a MVP-AT version 10 digitizer (Matrox Electronic Systems Limited, DORVAL, Quebec, Canada). The following set of filters was used: excitation $485 / 20 \mathrm{~nm}$, beamsplitter $510 \mathrm{~nm}$ and emission 515-565 nm (Zeiss, Oberkochen, Germany). The system was operated by the software package IMAGE1/AT $4.01 \mathrm{~b}$, (Universal Imaging Corporation, West Chester, Pa., USA).

Quantitation of fluorescence. After adjustment of black level, high voltage (550 keV) and gain (2.04) were adjusted such that the main part of the intensities (grey values) were within the midrange of the camera sensitivity. From each slide, in the $3 \times 3$ specifically labelled and in one background section, an area was randomly selected and an image was acquired by averaging and digitizing 16 video frames (PAL; 25 frames/s). The total illumination time was $1.5 \mathrm{~s}$. The resulting image $(512 \times 512$ pixels) was stored on a hard disk. After acquisition of the images, one dark and one white reference image were obtained using an uranyl-glass and 

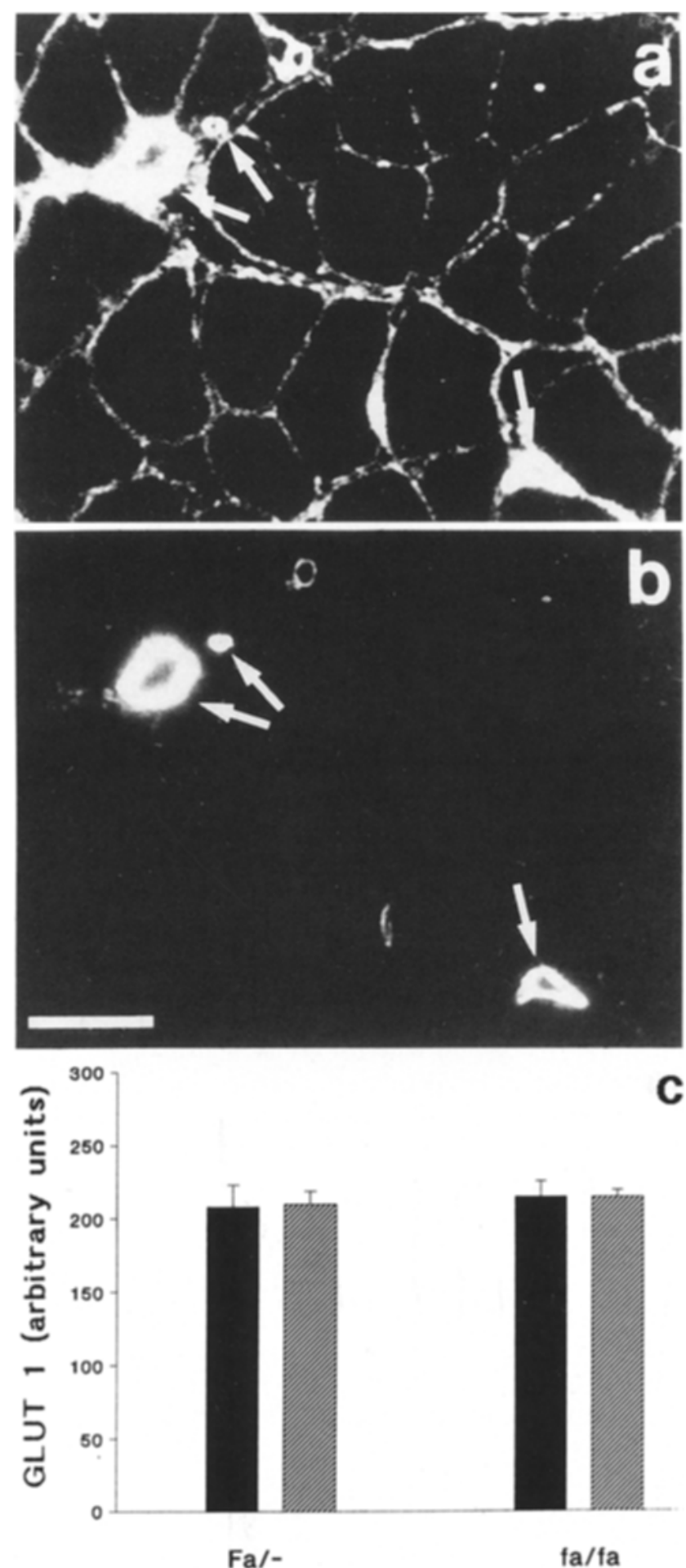

Fig.1a-c. GLUT 1 immunoreactivity in intramuscular perineurial sheaths. Cryosections of soleus muscles were immunolabelled with GLUT 1 antibody and BODIPY-conjugated secondary dntibody. a) Intense GLUT 1 immunoreactivity is present in the perineurial sheaths (arrows) and comparatively weak GLUT 1 immunoreactivity located near the sarcolemmal membrane is seen. The intensity of immunoreactivity was quantitated using images discriminated at a level higher than 60 grey values. b) The same image as a) after discrimination of immunofluorescence intensity higher than 60 grey values. Perineurial sheaths are again indicated (arrows) but the sarcolemmal signal is absent. Scale bar $75 \mu \mathrm{m}$. c) Mean of integrated intensities of GLUT 1 immunoreactivity are expressed in arbitrary units. $\mathrm{Fa} /$ - indicate control rats and fa/fa indicate obese, diabetic rats. Solid bars represent untreated rats and hatched bars represent rats treated with metformin for 24 days
Table 1. Characteristics of animals

\begin{tabular}{lcccc}
\hline & \multicolumn{2}{c}{$\mathrm{Fa} /{ }^{2}$} & \multicolumn{2}{c}{$\mathrm{fa} / \mathrm{fa}$} \\
Metformin & - & + & - & + \\
\hline$n$ & 7 & 6 & 7 & 5 \\
$\begin{array}{l}\text { Fasting } \\
\text { glucose } \\
\text { (mmol/l) }\end{array}$ & $6.4 \pm 0.3^{\mathrm{a}}$ & $6.6 \pm 0.3$ & $12.7 \pm 1.0^{\mathrm{c}}$ & $8.9 \pm 1.0$ \\
$\begin{array}{l}\text { Fasting } \\
\text { insulin } \\
\text { (pmol/l) }\end{array}$ & $2.8 \pm 0.6^{\mathrm{b}}$ & $1.6 \pm 0.5$ & $10.9 \pm 2.7^{\mathrm{c}}$ & $5.9 \pm 0.7$ \\
$\begin{array}{l}\text { Fructos- } \\
\text { amine }\end{array}$ & $155 \pm 8.4$ & $163.3 \pm 6.1$ & $186.8 \pm 12.6^{\mathrm{d}} 127.6 \pm 10.2$
\end{tabular}

${ }^{\mathrm{a}} p<0.002,{ }^{\mathrm{b}} p<0.01$ vs untreated fa/fa rats; ${ }^{\mathrm{c}} p<0.01,{ }^{\mathrm{d}} p<0.005$ vs metformin-treated fa/fa rats (one-sided Mann-Whitney).

Values are given as mean \pm SEM

images were corrected for background and shading [24]. The immunofluorescence was quantitated by collecting grey values higher than 60 as this signal represents immunoreactivity corresponding to the perineurial sheaths (Fig. 1). In each image the intensity in the undistorted part of the image area (3/4) was integrated. The sum of the integrated intensities from nine images were taken to represent a muscle.

Peroxidase-anti-peroxidase labelling. The localization of GLUT 1 in multiple sections from 4-10 different levels of the excised femoral nerve was visualized by the peroxidase-anti-peroxidase method, essentially as described in [6].

Morphometric analysis. Mean area fractions of perineurial sheaths in the BODIPY labeled sections of soleus muscle from each animal were determined as the area with grey value greater than 60 divided by the total image area. Mean area fractions of GLUT 1 immunoreactivity in peroxidase-anti-peroxidase labelled cross-sections of excised femoral nerves were determined by an analogous procedure and expressed as area of perineurial and endoneurial sheath divided by the total area of the nerve section.

\section{Statistical analysis}

Data in text, tables and figures are given as mean \pm SEM. Statistical significance between groups was tested by use of a MannWhitney test for unpaired comparisons, and Spearmans rho for correlation was used. One or two-sided tests were used where appropiate (indicated in Table 1). Statistical analysis was performed using MEDSTAT version 2.1, programmed by Drs. H. R. Wulff and P. Schlichting, Herlev University Hospital, Denmark.

\section{Results}

Pertinent values used for characterization of glucose homeostasis of animals are given in Table 1. The data presented in Table 1 have been previously reported[21].

The concentration of GLUT 1 in perineurial sheaths of intramuscular nerves and excised femoral nerves, respectively, was assessed by use of two different methods: 

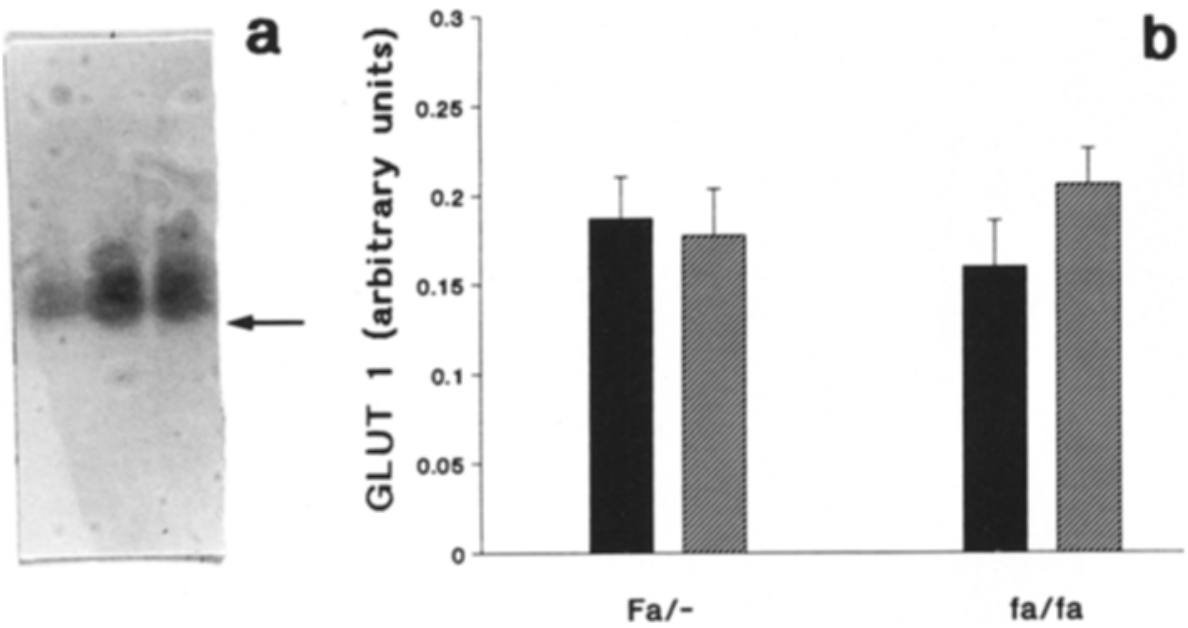

Fig. 2a,b. GLUT 1 immunoreactivity in perineurial sheaths of excised femoral nerves. Femoral nerves were excised and homogenates were subjected to SDSPAGE and immunoblotting. GLUT $1 \mathrm{im}-$ munoreactivity was determined by densitometry and results were corrected for the weight of excised nerve and area fraction of perineurial sheath in the nerves. a) Immunoblot of nerve-homogenate in three two-fold dilutions. Arrow indicate $45 \mathrm{kDa}$ molecular weight marker.b) GLUT 1 expression (arbitrary units) in control rats $(\mathrm{Fa} /-)$ and obese diabetic Zucker rats (fa/fa) without (solid bars) and with (hatched bars) treatment with metformin
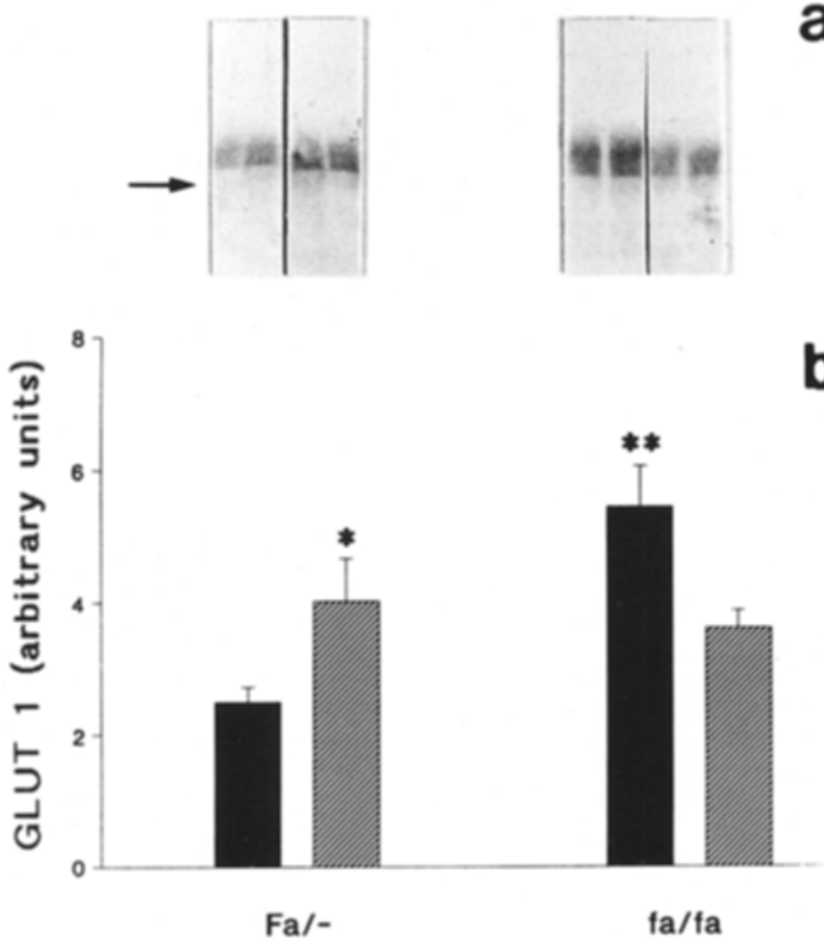

Fig.3a,b. GLUT 1 immunoreactivity of crude membranes from the soleus muscle. A crude membrane fraction was isolated from the soleus muscles and analysed by SDS-PAGE and immunoblotting, using GLUT 1 antibody as primary antibody. a) Representative immunoblots of crude membranes in duplicates from (from left to right) untreated and metformin treated control rats and diabetic (fa/fa) Zucker rats, respectively. Arrow indicates $45 \mathrm{kDa}$ molecular weight marker. b) Immunoreactivity was quantitated by densitometry and results were expressed relatively to protein concentration in the crude membranes. Solid bars, untreated rats and hatched bars, metformin-treated rats. ${ }^{*} p<0.025$ and ${ }^{* *} p<0.0025$ vs untreated control $(\mathrm{Fa} /-)$ rats

quantitative immunofluorescence (Fig. $1 \mathrm{a}, \mathrm{b}$ ) and immunoblotting of nerve homogenates (Fig. 2 a). Using quantitative immunofluorescence, we found no difference in mean GLUT 1 perineurial concentration in muscles from $(\mathrm{fa} / \mathrm{fa})$ rats compared with controls
(208.2 \pm 12.9 vs $203.3 \pm 11.4$ arbitrary units, NS) (Fig. 1c). Furthermore, mean perineurial GLUT 1 concentration in both groups was unaffected by metformin treatment (Fig. 1 c). The volume fraction of perineurial sheath in soleus muscle, determined morphometrically, was identical in the obese (fa/fa) Zucker rats without and with treatment with metformin and also when compared with the lean $(\mathrm{Fa} /-)$ controls without and with metformin treatment $(0.0023 \pm 0.0007 \%$ vs $0.0029 \pm$ $0.0006 \%$ vs $0.0020 \pm 0.0004 \%$ vs $0.0017 \pm 0.0006 \%$, respectively NS). When perineurial GLUT 1 concentration was determined in homogenates of excised femoral nerve by immunoblotting and densitometry, results were corrected group-wise for area fraction of perineurial sheaths in the dissected nerves, and expressed relative to the weight of nerve used for each preparation. GLUT 1 content in femoral nerves from the obese (fa/fa) rats was identical to the non-treated control $(\mathrm{Fa} /-)$ rats $(0.158 \pm 0.026$ vs $0.188 \pm 0.024$ arbitrary units, NS). Metformin did not influence GLUT 1 expression in either the obese $(\mathrm{fa} / \mathrm{fa})(0.158 \pm 0.026$ vs $0.205 \pm 0.020$ arbitrary units, NS) or in the control ( $\mathrm{Fa} /-$ ) rats $(0.187 \pm 0.024$ vs $0.177 \pm 0.026$ arbitrary units, NS) (Fig. 2b). Area fractions of perineurial sheaths in the four groups of animals studied were not significantly different $(0.110 \pm 0.011 \%$ vs $0.120 \pm 0.012 \%$ vs $0.124 \pm$ $0.023 \%$ vs $0.129 \pm 0.022 \%$, repectively).

GLUT 1 expression in crude membrane fractions of soleus muscles was quantitated by immunoblotting and densitometry (Fig. $3 \mathrm{a}$ ). In the untreated animals, GLUT 1 content was significantly higher in the fa/fa rats compared to the control group $(5.43 \pm 0.63$ vs $2.50 \pm 0.23$ arbitrary units, $p<0.005$ ) (Fig. $3 b$ ). Treatment with metformin significantly increased the GLUT 1 expression in the control $(\mathrm{Fa} /-)$ group $(2.50 \pm 0.23$ vs $4.01 \pm 0.65$ arbitrary units, $p<0.05)$ whereas in the obese $(\mathrm{fa} / \mathrm{fa})$ rats the GLUT 1 content tended to be decreased $(5.43 \pm 0.63$ vs $3.61 \pm 0.28$ arbitrary units, $p<0.075$ ) (Fig. $3 \mathrm{~b}$ ). Fasting glucose was positively correlated with GLUT 1 expression in crude 
membranes isolated from soleus muscle (rho $=0.44$, $p<0.05$ ) whereas fasting insulin was not correlated with GLUT 1 expression in soleus muscle. GLUT 4 expression was previously measured in crude muscle membranes of the soleus muscle from the same animals [21], and GLUT 1 and GLUT 4 expression was inversely correlated (rho $=0.52, p<0.01$ ).

\section{Discussion}

Among tissues present in rat skeletal muscle, GLUT 1 is expressed with the highest density in the perineurial sheaths and the endoneurial vessels of intramuscular nerves $[6,10-12]$. Recently we demonstrated that the muscle membranes proper contribute less than one-half of the total amount of GLUT 1 recovered in a crude membrane fraction purified from muscle [6]. Thus, identifying the source of the GLUT 1 in studies of its expression in skeletal muscle is necessary, since pure muscle cell membranes cannot be isolated. To circumvent this problem, we determined both the density of GLUT1 in perineurial sheaths of nerves from twodifferent localizations, and the total amount of GLUT 1 in a membrane fraction of skeletal muscle from the same animals. The density of perineurial GLUT 1 was normal in the moderately hyperglycaemic and hyperinsulinaemic, diabetic Zucker rats and unaffected by amelioration of diabetes by metformin treatment. Thus, unlike the situation in muscle cells in culture [25-27] and in various membrane fractions of muscle [28], the high-level perineurial expression of GLUT 1 was unaffected by hyperinsulinaemia and hyperglycaemia. The volume fractions of perineurial sheath in the soleus muscles were identical to those determined for Wistar rats in a previous study [6] and unaffected by both the fa/fa gene and the glucose homeostasis. Thereby the total amount of perineurial GLUT 1 in a given muscle is relatively constant and resistant to changes in glucose homeostasis.

The expression of GLUT 1 was furthermore determined in a crude membrane fraction isolated from homogenates of soleus muscles from the same experimental animals. We found an increased expression of GLUT 1 in the diabetic animals and the increase tended to be reduced following amelioration their of disease. As the perineurial contribution of GLUT 1 was constant, we have demonstrated that GLUT 1 expression in the diabetic fa/fa Zucker rats is preferably increased in the muscle cells. In a recent study, we have demonstrated a decreased expression of GLUT 4 in the same diabetic animals compared to lean littermates [21], indicating that a reciprocal regulation of GLUT 1 and GLUT 4 in skeletal muscle exists. Indeed, in muscles from all rats (i.e. including those treated with metformin), there was a negative correlation between GLUT 1 and GLUT 4 level. It could be speculated, that the increased GLUT 1 in the diabetic animals might partially compensate for the decreased GLUT 4 expression.
Whereas there is convincing evidence for a reciprocal regulation of GLUT 1 and GLUT 4 in adipocytes $[15,29]$ and cultured muscle cells $[26,27,30]$, earlier studies contain less information concerning regulation of transporters in skeletal muscle. Assuming that the present finding of a constant GLUT 1 level in perineurial sheaths can be applied on the various situations described below, the direction of the reported changes should be valid for the muscle cells proper. In agreement with our results, a differential affection of the expression of GLUT 1 and GLUT 4 in skeletal muscle is induced by denervation where GLUT 4 is decreased and GLUT 1 is increased [31]. Fasting and insulinopenic diabetes, however, affects GLUT 1 and GLUT 4 expression in rat skeletal muscle in parallel, both increase with fasting and decrease with diabetes $[10,32]$. It is, however, more relevant to compare our results on hyperinsulinaemic diabetic Zucker rats with studies performed under conditions where a certain insulin secretion capacity is preserved. A very recent study was designed to determine the role of circulating blood glucose levels on the number of glucose transporters in isolated membranes from normoinsulinaemic rats in the postabsorptive state. It was found that the effect of hyperglycaemia, in the absence of hypoinsulinaemia, is specific for glucose transporter isoform, with GLUT 1 and GLUT 4 varying in a reciprocal manner with hyperglycaemia [28]. The reciprocal regulation of the expression of the two transporters in obese diabetic Zucker rats proposed here, could also be mediated by plasma glucose. Thus, GLUT 1 correlated directly and GLUT 4 inversely [21] with fasting glucose. It has been suggested that an intracellular glucose metabolite is responsible for the reciprocal regulation of GLUT 4 and GLUT 1 expression [27]. The lack of effect of the increased plasma glucose on GLUT 1 expression in perineurial sheaths in the present fa/fa rats could be due to differences in glucose transport kinetics in perineurial sheaths and muscle cells. Such a difference could result from inherent kinetic differences between GLUT 1 and GLUT 4 , and could be amplified by postprandial insulin-induced recruitment of the latter. The amelioration of insulin resistance by metformin treatment of the diabetic rats, indicated by decreased fasting levels of glucose and insulin, could be achieved in part through the increased GLUT 1 expression in skeletal muscle which might increase glucose uptake in this tissue.

In conclusion, the expression of GLUT 1 in perineurial sheaths is unaffected by the fa/fa gene leading to obesity and insulin-resistant diabetes in the Zucker rat. Furthermore, amelioration of glucose homeostasis did not affect GLUT 1 expression in this tissue. In contrast, GLUT 1 in crude membranes isolated from the soleus muscle was increased in the diabetic rats, likely due to an increased expression in muscle cells, and it is proposed that GLUT 1 and GLUT 4 are differentially regulated in Zucker rats by plasma glucose. The design of future studies of glucose transporter levels in muscle 
should allow discrimination between GLUT 1 expression in perineurial sheath and in muscle membranes proper.

Acknowledgements. Ms. K. Clante and Ms. J. Harpøth are gratefully acknowledged for excellent technical assistance. This work was supported by the NOVO Foundation and the Danish Diabetes Association. The Velux Foundation of 1981 has donated the equipment for fluorescence microscopy. Dr. A. Handberg is the recipient of a research fellow grant from the NOVO Foundation.

\section{References}

1. Burant CF, Sivitz WI, Fukumoto H et al. (1991) Mammalian glucose transporters: structure and molecular regulation. Rec Prog Horm Res 47: 349-388

2. Pessin JE, Bell GI (1992) Mammalian facilitative glucose transporter family: structure and molecular regulation. Annu Rev Physiol 54: 911-930

3. Dick APK, Harik SI, Klip A, Walker DM (1984) Identification and characterization of the glucose transporter of the blood-brain barrier by cytochalasin $\mathrm{B}$ binding and immunological reactivity. Proc Natl Acad Sci USA 81: 7233-7237

4. Flier JS, Mueckler M, McCall AL, Lodish HF (1987) Distribution of glucose transporter messenger RNA transcripts in tissues of rat and man. J Clin Invest 79: 657-661

5. Mueckler M, Caruso C, Baldwin S et al. (1985) Sequence and structure of a human glucose transporter. Science 229: $941-$ 945

6. Handberg A, Kayser L, Høyer PE, Vinten J (1992) A substantial part of GLUT-1 in crude membranes from muscle originates from perineurial sheaths. Am J Physiol 262:E721-E727

7. Klip A, Ramlal T, Young DA, Holloszy JO (1987) Insulin-induced translocation of glucose transporters in rat hindlimb muscles. FEBS Lett 224: 224-230

8. Goodyear LJ, Hirshman MF, Smith RJ, Horton ES (1991) Glucose transporter number, activity, and isoform content in plasma membranes of red and white skeletal muscle. Am J Physiol 261:E556-E561

9. Douen AG, Ramlal T, Rastogi S et al. (1990) Exercise induces recruitment of the "insulin responsive glucose transporter". Evidence for distinct intracellular insulin- and exercise-recruitable transporter pools in skeletal muscle. J Biol Chem 265: 13427-13430

10. Kahn BB, Rosetti L, Lodish HF, Charron MJ (1991) Decreased in vivo glucose uptake but normal expression of GLUT 1 and GLUT 4 in skeletal muscle of diabetic rats. J Clin Invest 87: 2197-2206

11. Froehner SC, Davies A, Baldwin SA, Lienhard GE (1988) The blood-nerve barrier is rich in glucose transporter. J Neurocytol 17: 173-178

12. Harik SI, Kalaria RN, Anderson L, Lundahl P, Perry G (1990) Immunocytochemical localization of the erythroid glucose transporter: abundance in tissues with barrier functions. J Neurosci 10: 3862-3872

13. Crettaz M, Prentki M, Zanietti D, Jeanrenaud B (1980) Insulin resistance in soleus muscles from obese Zucker rats. Biochem J 186: 525-534

14. Sherman WM, Katz AL, Cutler CL, Withers RT, Ivy JL (1988) Glucose transport: locus of muscle insulin resistance in obese Zucker rats. Am J Physiol 255:E374-E382

15. Hainault I, Guerre-Millo M, Guichard C, Lavau M (1991) Differential regulation of adipose tissue glucose transporters in genetic obesity (fatty rat). J Clin Invest 87: 1127-1131
16. Zarjevski N, Doyle P, Jeanrenaud B (1992) Muscle insulin resistance may not be a primary etiological factor in the genetically obese fa/fa rat. Endocrinology 130: 1564-1570

17. Friedman JE, De Venté JE, Peterson RG, Dohm GL (1991) Altered expression of muscle glucose transporter GLUT-4 in diabetic fatty Zucker rats (ZDF/Drt-fa). Am J Physiol 261:E782-E788

18. Slieker LJ, Sundell KL, Heath WF et al. (1992) Glucose transporter levels in tissues of spontaneously diabetic Zucker fa/fa rat (ZDF/drt) and viable yellow mouse $\left(\mathrm{A}^{\mathrm{vy}}\right)$. Diabetes 41: 187-193

19. King PA, Horton ED, Hirshman MF, Horton ES (1992) Insulin resistance in obese Zucker rat (fa/fa) skeletal muscle is associated with a failure of glucose transporter translocation. J Clin Invest 90: 1568-1575

20. Friedman JE, Sherman WM, Reed MJ, Elton CW, Dohm GL (1990) Exercise training increases glucose transporter protein GLUT-4 in skeletal muscle of obese Zucker (fa/fa) rats. FEBS Lett 268: 13-16

21. Handberg A, Kayser L, Høyer PE, Voldstedlund M, Hansen HP, Vinten J (1993) Metformin ameliorates diabetes but does not normalize the decreased GLUT 4 content in skeletal muscle of obese (fa/fa) Zucker rats. Diabetologia 36:481-487

22. Handberg A, Vaag A, Damsbo P, Beck-Nielsen H, Vinten J (1990) Expression of insulin regulatable glucose transporters in skeletal muscle from type 2 (non-insulin-dependent) diabetic patients. Diabetologia 33: 625-627

23. Ploug T, Stallknecht BM, Pedersen O et al. (1990) Effect of endurance training on glucose transport capacity and glucose transporter expression in rat skeletal muscle. Am J Physiol 259:E778-E786

24. Jericevic Z, Wiese B, Bryan J, Smith LC (1989) Validation of an imaging system: steps to evaluate and validate a microscope imaging system for quantitative studies. In: Taylor DL, Wang Y-L (eds); Methods in cell biology. Academic Press, New York, pp 47-83

25. Mayor P, Maianu L, Garvey WT (1992) Glucose and insulin chronically regulate insulin action via different mechanisms in $\mathrm{BC}_{3} \mathrm{H} 1$ myocytes. Effects on glucose transporter gene expression. Diabetes 41:274-285

26. Mitsumoto Y, Burdett E, Grant A, Klip A (1991) Differential expression of the GLUT 1 and GLUT 4 glucose transporters during differentiation of L6 muscle cells. Biochem Biophys Res Commun 175: 652-659

27. Koivisto U-M, Martinez-Valdez H, Bilan PJ, Burdett E, Ramlal T, Klip A (1991) Differential regulation of the GLUT-1 and GLUT-4 glucose transport systems by glucose and insulin in L6 muscle cells in culture. J Biol Chem 266: 2615-2621

28. Dimitrakoudis D, Ramlal T, Rastogi S, Vranic M, Klip A (1992) Glycemia regulates the glucose transporter number in the plasma membrane of rat skeletal muscle. Biochem J 284: 341-348

29. Pedersen O, Kahn CR, Kahn BB (1992) Divergent regulation of the GLUT 1 and GLUT 4 glucose transporters in isolated adipocytes from Zucker rats. J Clin Invest 89: 1964-1973

30. Bashan N, Burdett E, Hundal HS, Klip A (1992) Regulation of glucose transport and GLUT 1 glucose transporter expression by $\mathrm{O}_{2}$ in muscle cells in culture. Am J Physiol 262:C682-C690

31. Block NE, Menick DR, Robinson KA, Buse MG (1991) Effect of denervation on the expression of two glucose transporter isoforms in rat hindlimb muscle. J Clin Invest 88: 1546-1552

32. Kahn BB (1992) Facilitative glucose transporters: regulatory mechanisms and dysregulation in diabetes. J Clin Invest 89: 1367-1374 